\title{
PROPHYLAXIS AGAINST BLENNORRHOEA NEONATORUM IN DENMARK*
}

\author{
BY
}

\section{GORDON NORRIE}

COPENHAGEN

CREDÉ's method was adopted in the Royal Lying-in Hospital of Copenhagen in the year 1882, and on the basis of the results obtained two different papers were read at the International Medical Congress held at Copenhagen in 1884. In 1886, I began a series of papers, chiefly in the medical press, with the object of having the method made compulsory for midwives. In this I was successful in 1900 , and since that date it has been compulsory for every midwife to instil a solution of silver nitrate $(1: 150)$ into the eyes of the child. Before doing so the eyes must not be washed but are to be wiped with dry cotton or gauze.

I may remark (1) that midwives assist at almost every delivery in Denmark and that it is very seldom that only a doctor is present; (2) that since 1714 the midwifery system is legally regulated; (3) that a very severe training school for midwives has been in existence since 1787 ; and (4) that the time of training for midwives is one year.

Before 1857 the education of blind children was in private hands, but in that year the state erected the Royal Blind Institute (Det Kongelige Blindeinstitut) in Copenhagen. Although it is not compulsory for blind children to go into the Institute, there is scarcely any blind child educated outside it, i.e. practically all blind children are to be found in it. The age of admission into the Institute was originally from ten to eleven, but as the Institute had not sufficient room to receive all the blind children, a preparatory school (Den Kongelige Blindeforskole) lying near the sea and about 60 miles from Copenhagen was established. In this school the children are accepted from their sixth to seventh year. Some two years ago at the same place and under the same director an asylum for small blind children was also established. The youngest patient at present under treatment there was born in 1920 and is blind as a result of ophthalmia neonatorum. I hold the posts of doctor for the Copenhagen Institute and oculist for the preparatory school.

In the following table I give the numbers of children blind as a result of ophthalmia neonatorum in both Danish Institutes arranged according to the year of their birth in order to show the results of prophylaxis. Of course it is not to be expected that any great improvement would follow immediately on the introduction of prophylactic methods as time had to be allowed for the midwives to

\footnotetext{
* We are indebted to the Ministry of Health for this valuable communication.
} 
learn the necessary procedure. The table shows that there is no change for the first five years, but that after 1905 the result is very evident.

Of children born 1875-1879 12 were blind from oph. neonat.

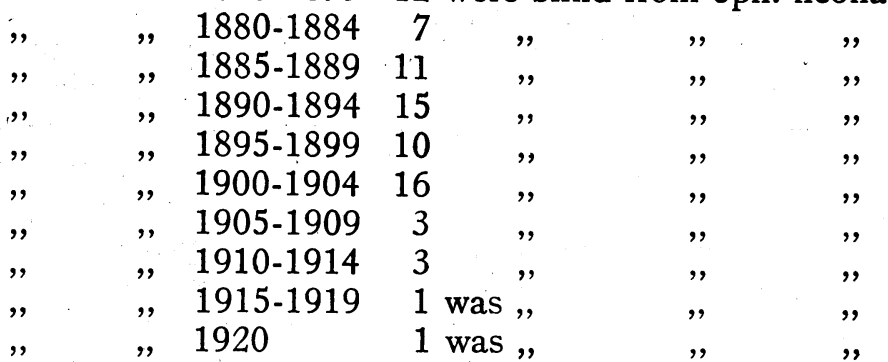

Practically all blind children born before 1916 are in the Institutes ; some children, however, born since that year may still remain in their homes, and some of these may owe their blindness to ophthalmia neonatorum.

Thanks to the excellence of our midwives our results seem to show that we may only expect one case every second year. We may possibly even improve on this, but it must be remembered that prophylactic measures cannot prevent a later infection after birth.

I may add that the population of Denmark is $3,250,000$.

\section{ANNOTATIONS}

\section{Standards for Out-Patient Service in Ophthalmology}

An interesting report on this subject is issued in the April number of the American Journal of Ophthalmology by the Ophthalmological section of the Associated Out-Patient Clinics of New York City. The report is divided into two sections: (1) a summary of conditions found by survey of representative eye clinics in New York; (2) an outline for an ideal out-patient service in Ophthalmology. The first part deals with the subject under the following headings: consultation facilities; medical organization; space, arrangement, equipment and facilities; admission and distribution of patients; fees ; routine history and examination; refraction and provision of glasses; follow up; records and filing; research and teaching; efficiency tests and statistics. Probably the most interesting portion of this section is that dealing with refraction, in that the reporters advise that refraction work be paid for. No ophthalmic surgeon will question the advisability of this step; it is ideal, but not always possible to obtain, at any rate in this country at the present time; nor will any deny the supreme 